Particle Accelerators, 1995, Vol. 51, pp. 15-28

Reprints available directly from the publisher

Photocopying permitted by license only (c) 1995 OPA (Overseas Publishers Association) Amsterdam B.V. Published under license by Gordon and Breach Science Publishers SA Printed in Malaysia

\title{
MICROWAVE INSTABILITIES IN ELECTRON RINGS WITH NEGATIVE MOMENTUM COMPACTION FACTOR
}

\author{
S.X. FANG, ${ }^{*}$ K. OIDE, K. YOKOYA, B. CHEN ${ }^{\dagger}$ and J.Q. WANG* \\ KEK, National Laboratory for High Energy Physics \\ Oho, Tsukuba, Ibaraki, 305 Japan \\ (Received 10 February 1995; in final form 12 May 1995)
}

\begin{abstract}
Bunch lengthening (or shortening) caused by the potential well distortion and the microwave instability in electron rings with negative momentum compaction factor is discussed in detail based on the resonator impedance model; further, a comparison with rings of positive momentum compaction factor is given. It was found that the bunch shape is less deformed and the current threshold of the microwave instability is higher in the rings with negative momentum compaction factor over a very wide range of the impedance parameters. The results also show that even within the range where the threshold for positive momentum compaction is higher than that for negative momentum compaction, the bunch lengthening is still less serious in most cases. The main reason is that the beam is less deformed by its own wake field, and some "negative feedback" mechanism plays an important role in rings with negative momentum compaction factor. Finally, an example of bunch lengthening in the case $\alpha<0$ with a real wake field of the old chamber of the SLC damping ring is given in contrast to the case in which $\alpha>0$.
\end{abstract}

KEY WORDS: Microwave instabilities, momentum compaction factor, bunch lengthening

\section{INTRODUCTION}

The bunch length variation due to the microwave instability is a very interesting problem for electron-positron colliders; ${ }^{1}$ especially, in most of the existing low energy electronpositron colliders, a positive momentum compaction $(\alpha>0)$ is usually adopted. Bunch lengthening in such machines is one of the most important factors which limits the luminosity performance and its upgrade.

The idea to understand the bunch lengthening phenomenon in rings with negative momentum compaction factor $(\alpha<0)$ was initiated by the desire to upgrade the luminosity of BEPC (Beijing Electron Positron Collider). ${ }^{2}$ For further luminosity upgrading, several

\footnotetext{
* On leave of absence from Institute of High Energy Physics, Academia Sinica, Beijing, China.

${ }^{\dagger}$ Superconducting Super Collider Laboratory, Dallas.
} 
approaches were proposed, among which mini- $\beta$ insertion was the most promising. However, the crucial factor for the BEPC mini- $\beta$ is the bunch length. At present, the average maximum luminosity of BEPC is $6 \times 10^{30} \mathrm{~cm}^{2} \mathrm{sec}^{-1}$ at $2.0 \mathrm{GeV}$; the corresponding bunch length according to the measurement is about $7-8 \mathrm{~cm}$, which is about twice as large as the calculated value $(4.16 \mathrm{~cm})$. In order to shorten the bunch length from $7 \mathrm{~cm}$ to $\sim 3 \mathrm{~cm}$, so as to fit the requirement of a $3.6 \mathrm{~cm}$ mini- $\beta$ configuration, by increasing the RF voltage from $1 \mathrm{MV}$ to $2.4 \mathrm{MV}$, two more cavities, (provided by the CERN SPS division) have been added to the BEPC ring. However, recent experimental results have shown that bunch shortening by increasing the RF voltage is less than the value predicted by theory. According to the scaling law of BEPC, obtained by the bunch length measurement, ${ }^{3}$ the bunch length is still about $4.5 \mathrm{~cm}$ at $2.0 \mathrm{GeV}, 50 \mathrm{~mA}$, with a $2.4 \mathrm{MV}$ RF voltage. Hence, finding a different approach to shorten the bunch length becomes a very important issue. Using negative momentum compaction is one of the hopeful approaches. ${ }^{4}$

In this paper, the method and computer program developed by K. Oide and K. Yokoya ${ }^{5}$ are used to study the longitudinal bunch behaviour. First, the equation of motion is reviewed simply. Then, the potential well distortion (PWD) and the threshold of microwave instabilities with a resonator impedance model for both the $\alpha>0$ and $\alpha<0$ cases are discussed and compared in detail in the second and third sections. Finally, as an example, bunch lengthening in a ring with a real wake field of the old chamber of the SLC damping ring for $\alpha>0$ and $\alpha<0$ is demonstrated. Generally speaking, bunch lengthening is less serious in rings with $\alpha<0$.

All of the above results will not only be beneficial in upgrading the luminosity of BEPC, but will also be useful for future high-luminosity collider designing.

\section{EQUATION}

The canonical variables used are the normalized energy deviation $\left(p=\frac{-\delta}{\sigma_{E 0}}\right)$ and normalized longitudinal distance ( $q=\frac{z}{\sigma_{z 0}}$ ) from the synchronous particle. Here, $\eta$ is the slippage factor, $\sigma_{z 0}$ the rms natural bunch length and $\sigma_{E 0}$ the r.m.s. natural energy spread.

The single particle motion is described by the Hamiltonian $(H)$ as

$$
H=\frac{1}{2} p^{2}+\frac{1}{2} q^{2}-I_{k, s} \int_{q}^{\infty} \int_{q^{\prime}}^{\infty} W_{N}\left(q^{\prime}-q^{\prime \prime}\right) \rho\left(q^{\prime \prime}\right) d q^{\prime \prime} d q^{\prime} .
$$

Here, $W_{N}(q)$ is the normalized wake field, which is generated by the particles in the bunch, and is equal to zero in the region $q>0$ by causality. $\rho(q)$ is the particle density in the bunch, and $I_{k, s}$ is the well-known dimensionless Keil-Schnell criterion parameter,

$$
I_{k, s}=\frac{I R_{s}}{\eta \sigma_{E 0}^{2}\left(\frac{E}{e}\right)} .
$$

$I$ is the peak current, $R_{S}$ the shunt impedance of a broad-band resonator, and $\frac{E}{e}$ the nominal beam energy in unit of Volts. 
The relation between the normalized longitudinal wake field $\left(W_{N}(q)\right)$ and the usual wake-field $\left(W_{\|}(q)\right)$ is

$$
W_{N}(q)=\frac{\sigma_{z 0}}{c R_{S}} W_{\|}(q)
$$

For a resonator wake field,

$$
W_{N}(q)=\frac{K}{Q} \exp \left(\frac{K}{2 Q} q\right) \sin \left(\sqrt{4 Q^{2}-1} \frac{K}{2 Q} q+\tan ^{-1}\left(\sqrt{4 Q^{2}-1}\right)\right) \text {, for } Q>0.5, q<0
$$

Here, $K=\omega_{r} \frac{\sigma_{z 0}}{c}, \omega_{r}$ is the resonator frequency, and $c$ is the speed of light. Thus, $W_{\|}(q)$ is fully determined by only two parameters, $K$ and $Q$. The corresponding equations of motion are:

and

$$
\frac{d q}{d s}=\frac{\partial H}{\partial p}=p
$$

$$
\frac{d p}{d s}=-\frac{\partial H}{\partial q}=-q-I_{k, s} \int_{q}^{\infty} W_{N}\left(q-q^{\prime}\right) \rho\left(q^{\prime}\right) d q^{\prime},
$$

where $s=\omega_{s 0} t, \omega_{s 0}$ is the unperturbed frequency of the synchrotron motion, and $s$ is the phase of the synchrotron motion. From Eq. (2), the following Vlasov equation is obtained:

$$
\frac{\partial \psi}{\partial s}+p \frac{\partial \psi}{\partial q}-q \frac{\partial \psi}{\partial p}-I_{k, s} \frac{\partial \psi}{\partial p} \int_{q}^{\infty} W_{N}\left(q-q^{\prime}\right) \rho\left(q^{\prime}\right) d q^{\prime}=2 \lambda_{0} \frac{\partial}{\partial p}\left(\frac{\partial \psi}{\partial p}+p \psi\right)
$$

$\psi=\psi(p, q, s)$ is the distribution function in phase space $(p, q)$, where $\lambda_{0}=\frac{1}{\tau_{0} \omega_{s 0}}, \tau_{0}$ being the radiation damping time and $\rho\left(q^{\prime}\right)=\int \psi\left(p, q^{\prime}\right) d p$.

Splitting $\psi(p, q, s)$ into two parts,

$$
\psi(p, q, s)=\psi_{0}(p, q)+\psi_{1}(p, q, s)
$$

and ignoring the nonlinear terms in $\psi_{1}$, we obtain the following linearized Vlasov equation for $\psi_{1}$ :

$$
\begin{aligned}
& \frac{\partial \psi_{1}}{\partial s}+p \frac{\partial \psi_{1}}{\partial q}-q \frac{\partial \psi_{1}}{\partial p}-I_{k, s} \frac{\partial \psi_{0}}{\partial p} \int_{q}^{\infty} \int_{-\infty}^{\infty} d p^{\prime} d q^{\prime} W_{N}\left(q-q^{\prime}\right) \psi_{1}\left(p^{\prime}, q^{\prime}, s\right) \\
= & 2 \lambda_{0} \frac{\partial}{\partial p}\left(\frac{\partial \psi_{1}}{\partial p}+p \psi_{1}\right) .
\end{aligned}
$$


$\psi_{0}(p, q)$ is a stationary solution of the Haissinski equation,

$$
p \frac{\partial \psi_{0}}{\partial q}-q \frac{\partial \psi_{0}}{\partial p}-I_{k, s} \frac{\partial \psi_{0}}{\partial p} \int_{q}^{\infty} \int_{-\infty}^{\infty} d p^{\prime} d q^{\prime} W_{N}\left(q-q^{\prime}\right) \psi_{0}\left(p^{\prime}, q^{\prime}\right)=0 .
$$

By transforming $(p, q)$ to action-angle variables $(J, \phi),{ }^{6}$ the Vlasov equation can be finally rewritten as

$$
\begin{aligned}
\frac{\partial \psi_{1}}{\partial s}= & -\omega(J) \frac{\partial \psi_{1}}{\partial \phi}+\left.I_{k, s} \frac{\partial \psi_{0}}{\partial J} \frac{\partial}{\partial \phi}\right|_{J} \int_{J} \int_{\phi} U\left(q(J, \phi)-q^{\prime}\left(J^{\prime}, \phi^{\prime}\right)\right) \psi_{1}\left(J^{\prime}, \phi^{\prime}, s\right) d J^{\prime} d \phi^{\prime} \\
& +2 \lambda_{0} \frac{\partial}{\partial J}\left(J\left(\psi_{1}+\frac{1}{\omega(J)} \frac{\partial \psi_{1}}{\partial J}\right)\right)
\end{aligned}
$$

where, $U(q)=\int_{-\infty}^{q} W(q) d q, \omega(J)$ is the incoherent synchrotron oscillation frequency spread and $\omega(J)=\frac{\partial H(J)}{\partial J}$.

Eq. (6) is an integral equation with an intensity dependent term and a frequency spread term on the right side, which are not easy to deal with. However, using the method and computer program developed by K. Oide and K. Yokoya, ${ }^{5}$ one can easily obtain a numerical solution; the convergence is good up to $I_{k, s} \sim 20$ for various $Q$ and $K<2$ of the resonator impedance model.

\section{POTENTIAL WELL DISTORTION}

When the beam intensity is not sufficiently high, i.e., below the microwave threshold, the bunch behaviour is determined by its own potential well field. The bunch shape is deformed and its length is changed. A self-consistent solution of the bunch shape is found by applying numerical integration of the Haissinski equation (5), or using the more general approach of solving a truncated coupled set of non-linear equations. ${ }^{7}$ The results obtained by these two methods agree well with the closed analytic form of the pure resistive impedance and the pure inductive impedance for both the $\alpha>0$ and $\alpha<0$ cases.

For the resonator impedance model, the typical distribution of the particles in the bunch vs current is given in the Figures 1 (a) and 1 (b) for $Q=1$ and $K=1$. One can see that the cases $\alpha>0$ and $\alpha<0$ are quite different.

1. As usual, the bunch leans forward to compensate for the energy loss by the RF voltage in the case $\alpha>0$, and backward for $\alpha<0$.

2. The bunch shape is seriously distorted for $\alpha>0$; for high intensity, sometimes two peaks appear. However, it is less distorted for $\alpha<0$.

As indicated in Fig. 2, the reason is obvious: in the case $\alpha>0$, most particles move to the front part of the bunch; then, the wake field produced by them disturbs the tail part of 


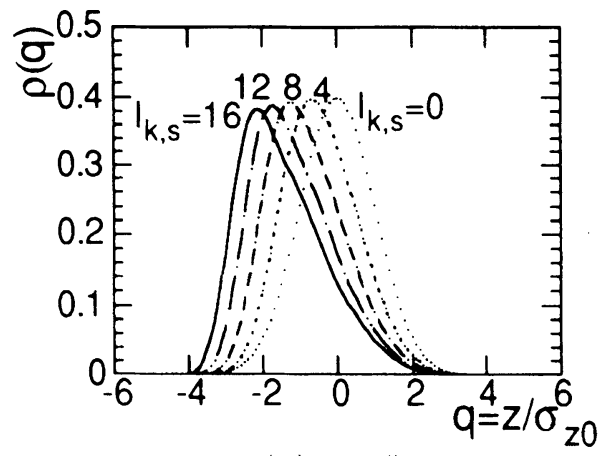

(a) $n<0$

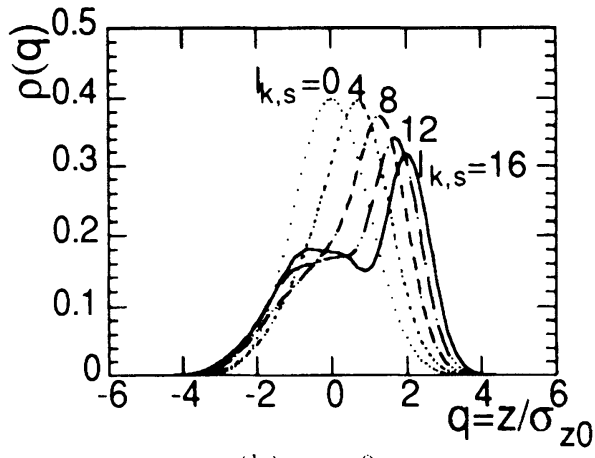

(b) $a>0$

FIGURE 1: Bunch shape (density distribution within the bunch) for various intensities. (In the case of $\alpha<0$, the absolute value of $I_{k, s}$ is indicated.)

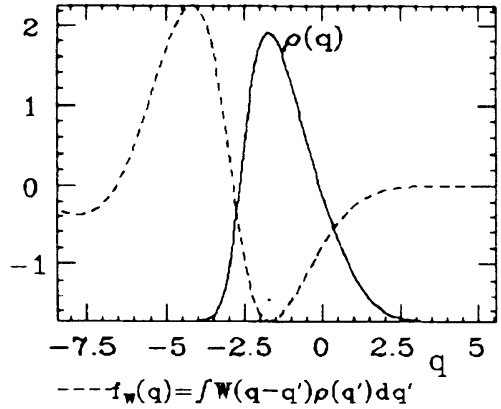

(a) $a<0$

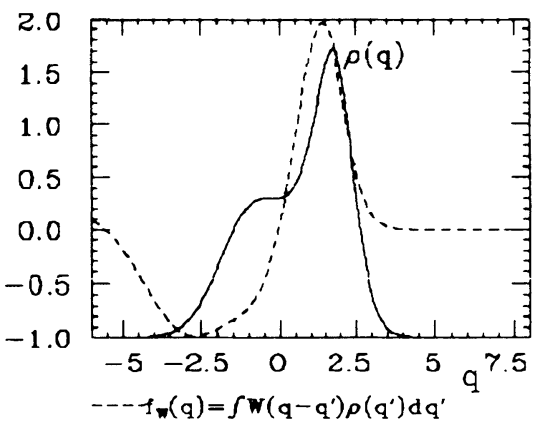

(b) $a>0$

FIGURE 2: Relative location (the solid line) of the bunch and its wake field (the dashed line).

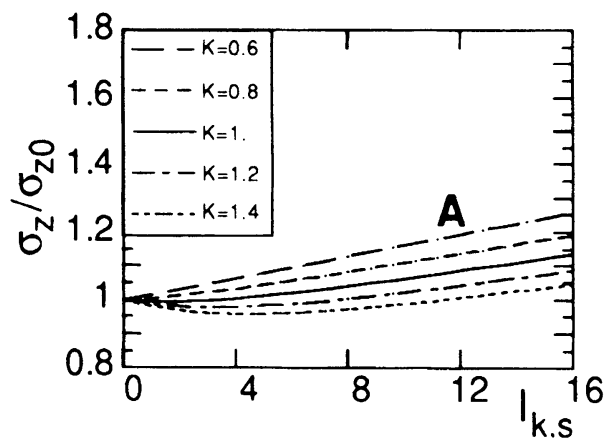

(a) $n<0$

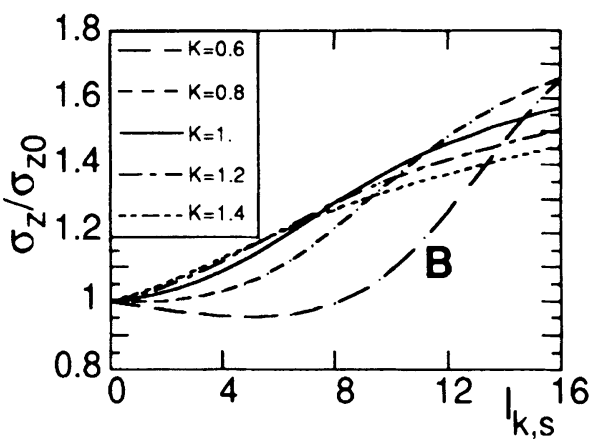

(b) $n>0$

FIGURE 3: Bunch length vs current, $Q=1$. 
the beam, and a large deformation occurs at the tail part at high intensity. However, in the case $\alpha<0$, the situation is different: most particles move to the back of the bunch; since the wake field generated by these particles is mostly located outside of the beam, only a portion of the beam is disturbed. A calculation shows that two peaks never appear in the $\alpha<0$ case, and that the beam always remains in good order.

Figure 2(b) shows that the minimum of the wake field is near to the second peak of the bunch, and that it accelerates the tail part of the beam.

Another very interesting phenomenon is the bunch length variation vs the current (Figures 3(a) and 3(b)).

Bunch lengthening is very serious in the case $\alpha>0$; at high intensity, ${ }^{a}$ the lengthening factor is between 1.45 to 1.65 . However, it is between 1.05 to 1.25 for $\alpha<0$. Although Figures 3(a) and 3(b) are only for the case $Q=1$, a similar phenomenon occurs for different $Q$. This can be explained as follows.

For a long bunch $(K>1)$, in the case $\alpha>0$, the spectrum is mainly in the inductive part of the impedance, which causes the bunch to lengthen and moves the spectrum even further to the inductive part of the impedance. It is like a "positive feedback", which causes the bunch length to increase exponentially with the intensity. However, in the case $\alpha<0$, most of the spectrum of a long bunch is in the negative-inductive part of the impedance, which causes the bunch to shrink, thus forming a "negative feedback". As a result, the bunch length increase is nearly linear and very slight.

For a short bunch $(K<1)$, the situation is similar in the case $\alpha<0$, in that most of the spectrum of the short bunch overlaps with the negative-capacitive part of the impedance, which causes bunch lengthening. The spectrum thus moves to the negativeinductive part of the impedance, which partly cancels the negative-capacitive part and a "negative feedback" mechanism is formed, so that the lengthening becomes weaker. This is why bunch lengthening of a short bunch is always mild, as shown in Figure 3(a), curve "A". However, it is quite different in the case $\alpha>0$; it seems there should be a "positive feedback" mechanism to push the bunch length so as to become shorter. However, it is not true when the bunch is shortened to some extent; in this case the wake field generated by the head part of the bunch is so strong that it causes a serious deformation of its tail part. The bunch shortening is then stopped and lengthening starts. Once the bunch becomes lengthened, "positive feedback" plays an important role, and the bunch length increases even more significantly, due to the fact that the intensity is already very high at that time. This can be seen from the curve "B" in Figure 3(b).

\section{MICROWAVE INSTABILITY}

Above the current threshold of the microwave instability, one can find a numerical solution for the Vlasov equation (Eq. (6)) by putting the Haissinski solution $\left(\psi_{0}(J)\right)$ of potential well distortion in it. The main results for the resonator impedance model with various parameters are described as follows:

\footnotetext{
${ }^{a}$ For example, $I_{k, s}=16$ corresponds to $40 \mathrm{~mA}$ for BEPC
} 
1. In the case $\alpha<0$, the mechanism of microwave instabilities is mainly due to radial mode coupling within a single azimuthal band; in the case that $\alpha>0$, however, besides the radial mode coupling belonging to the same azimuthal band, instabilities are sometimes excited by the combined function of radial and azimuthal mode coupling. The reason can be explained qualitatively. Let's expand $\psi_{1}(J, \phi, s)$ in terms of eigenfunctions:

$$
\psi_{1}(J, \phi, s)=\sum_{m=-\infty, m \neq 0}^{m=\infty} R_{m}(J) e^{i m \phi} \cdot e^{-i \Omega s} .
$$

Here, $m$ is the number of azimuthal modes and $\Omega=\frac{\omega_{s}}{\omega_{s 0}}$ is the coherent frequency in units of $\omega_{s 0}$. Substituting Eq. (7) into Eq. (6) and multiplying by $e^{-i m \phi}$ on both sides and then integrating over $\phi$, we obtain

$$
-i(\Omega-m) R_{m}(J)-i m I_{k, s} \frac{\partial \psi_{0}}{\partial J} \int \sum_{m^{\prime}} G_{m m^{\prime}}\left(J, J^{\prime}\right) R_{m^{\prime}}\left(J^{\prime}\right) d J^{\prime}=0 .
$$

In order to discuss only the azimuthal mode, the dependence of $\omega(J)$ on $J$ and the corresponding radial mode can be neglected; thus, we take $\omega(J)=1$, i.e., without any frequency spread. The radiation damping term in Eq. (6) is also neglected for simplicity. Here,

$$
G_{m m^{\prime}}\left(J, J^{\prime}\right)=\frac{1}{2 \pi} \oint d \phi e^{-i m \phi} \oint d \phi^{\prime} e^{i m^{\prime} \phi^{\prime}} U\left(q(J, \phi)-q\left(J^{\prime}, \phi^{\prime}\right)\right) .
$$

Substituting the Fourier transform of $U(q)$ into Eq. (9),

$$
U(q)=\frac{1}{2 \pi} \int_{-\infty}^{\infty} \tilde{U}\left(\tilde{\omega} \frac{\sigma_{z 0}}{c}\right) \exp \left(-i \tilde{\omega} \frac{\sigma_{z 0}}{c} q\right) d \tilde{\omega}
$$

and

$$
\tilde{U}(\tilde{\omega})=\int_{-\infty}^{\infty} U(q) \exp \left(i \tilde{\omega} \frac{\sigma_{z 0}}{c} q\right) \frac{\sigma_{z 0}}{c} d q=\frac{i Z(\tilde{\omega})}{\tilde{\omega}+i 0},
$$

where, $Z(\tilde{\omega})$ is the resonator impedance, we get

$$
G_{m m^{\prime}}\left(J, J^{\prime}\right)=i \int_{-\infty}^{\infty} d \tilde{\omega} \frac{Z(\tilde{\omega})}{\tilde{\omega}+i 0} F_{m}^{\star}(\tilde{\omega}, J) F_{m^{\prime}}\left(\tilde{\omega}, J^{\prime}\right),
$$

with

$$
F_{m}(\tilde{\omega}, J)=\frac{1}{2 \pi} \oint d \phi \exp \left(i m \phi+i \tilde{\omega} \frac{\sigma_{z 0}}{c} q(\phi, J)\right) .
$$

Note that, for low intensity, $q=\sqrt{2 J} \cos \phi$, and thus

$$
F_{m}(\tilde{\omega}, J)=i^{-m} J_{m}\left(\omega \frac{\sigma_{z 0}}{c} \sqrt{2 J}\right)
$$

where $J_{m}$ is the Bessel function. 
One can introduce the spectrum of the bunch $\left(\sigma_{m}(\tilde{\omega})\right)$ as

$$
\sigma_{m}(\tilde{\omega})=\int F_{m}(\tilde{\omega}, J) R_{m}(J) d J .
$$

Multiplying by $F_{m}\left(\tilde{\omega}^{\prime}, J\right)$ on both sides of Eq. (8) and integrating over $J$, we finally obtain

$$
\sum_{m} \sigma_{m}(l)=\sum_{p=-\infty}^{\infty} i \frac{Z(p)}{p} M_{l p} \sum_{m^{\prime}} \sigma_{m^{\prime}}(p)
$$

where

$$
M_{l p}=\sum_{m=-\infty, m \neq 0}^{\infty} \frac{m}{\Omega-m} I_{k, s} \int-\frac{d \psi_{0}}{d J} F_{m}^{\star}(p, J) F_{m}(l, J) d J
$$

is the matrix element.

Here, $p$ and $l$ represent $\tilde{\omega}=p \omega_{0}+\Omega$, and $\tilde{\omega}^{\prime}=l \omega_{0}+\Omega$, respectively; $\omega_{0}$ is the revolution frequency and $\int \frac{Z(\tilde{\omega})}{\tilde{\omega}}$ is replaced by $\sum_{p=-\infty}^{\infty} \frac{Z(p)}{p}$.

For an analysis, only two adjacent modes $(m$ and $m+1)$ are considered. We assume that the impedance has a very simple form, as shown in Figure 4; its resonant frequency $\left(\omega_{r}=p_{r} \omega_{0}+\Omega \omega_{s 0}\right)$ lies just between the maximum of the two spectrum modes ( $m$ and $m+1)$.

From Figure 4, one can see that the $\sigma_{m}$ spectrum overlaps with the inductive part of the impedance while the $\sigma_{m+1}$ spectrum overlaps with the capacitive part. Thus, for $p=p_{r}$,

$$
\begin{aligned}
\sigma_{m}\left(p_{r}\right)+\sigma_{m+1}\left(p_{r}\right) \cong & I_{k, s} \sum_{p=p_{r},-p_{r}} i \frac{Z(p)}{p} M_{p_{r}, p}\left(\sigma_{m}(p)+\sigma_{m+1}(p)\right) \\
= & i \frac{R_{s}}{p_{r}} M_{p_{r}, p_{r}}\left(\sigma_{m}\left(p_{r}\right)+\sigma_{m+1}\left(p_{r}\right)\right) \\
& -i \frac{R_{s}}{p_{r}} M_{p_{r},-p_{r}}\left(\sigma_{m}\left(-p_{r}\right)+\sigma_{m+1}\left(-p_{r}\right)\right) \\
& +\frac{L}{p_{r}} M_{p_{r}, p_{r}} \sigma_{m}\left(p_{r}\right)+\frac{L}{p_{r}} M_{p_{r},-p_{r}} \sigma_{m}\left(-p_{r}\right) \\
& -\frac{C}{p_{r}} M_{p_{r}, p_{r}} \sigma_{m+1}\left(p_{r}\right)-\frac{C}{p_{r}} M_{p_{r},-p_{r}} \sigma_{m+1}\left(-p_{r}\right) .
\end{aligned}
$$

A similar relation can be obtained for $p=-p_{r}$.

In the above expression

$$
M_{p_{r}, \pm p_{r}}=\frac{m}{\Omega-m} I_{k, s} \mathcal{L}_{m}\left(p_{r}, p_{r}\right) \pm \frac{m+1}{\Omega-(m+1)} I_{k, s} \mathcal{L}_{m+1}\left(p_{r}, p_{r}\right),
$$

here, $\mathcal{L}_{k}(k=m, m+1)$ is $\mathcal{L}_{k}\left(p_{r}, \pm p_{r}\right)=\int-\frac{\partial \psi_{0}}{\partial J} F_{k}\left(p_{r}, J\right)^{*} F_{k}\left( \pm p_{r}, J\right) d J$ is a positive quantity due to the fact that $-\frac{\partial \psi_{0}}{\partial J}$ is positive. 


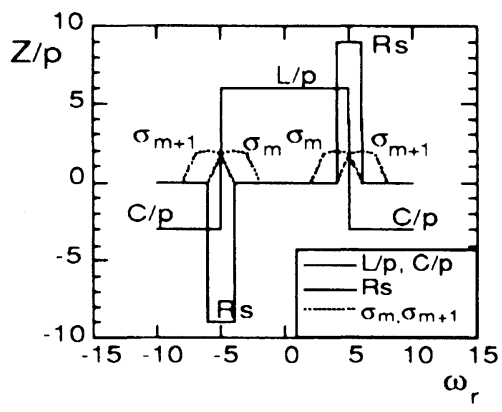

$$
\begin{array}{llll}
i \frac{Z(p)}{p}= \pm i \frac{R_{s}}{p_{r}} & \text { at } & p= \pm p_{r} . \\
i \frac{Z(p)}{p}=\frac{L}{p_{r}} & \text { when } & |p|<\left|p_{r}\right| . \\
i \frac{Z(p)}{p}=-\frac{C}{p_{r}} & \text { when } & |p|>\left|p_{r}\right| .
\end{array}
$$

FIGURE 4: Simplified resonator impedance model.

Notice that $\sigma_{m}\left(-p_{r}\right)=(-1)^{m} \sigma_{m}\left(p_{r}\right)$, and that $\mathcal{L}_{m}\left(p_{r},-p_{r}\right)=\mathcal{L}_{m}\left(p_{r}, p_{r}\right), \mathcal{L}_{m+1}\left(p_{r},-p_{r}\right)$ $=-\mathcal{L}_{m+1}\left(p_{r}, p_{r}\right)$, when $m$ is even. Then, the matrix equation reduces to

$$
\left(\begin{array}{c}
\sigma_{m}\left(p_{r}\right) \\
\sigma_{m+1}\left(p_{r}\right)
\end{array}\right)=2 I_{k, s}\left(\begin{array}{cc}
\frac{L}{p_{r}} \frac{m \mathcal{L}_{m}}{\Omega-m} & i \frac{R_{s}}{p_{r}} \frac{m \mathcal{L}_{m}}{\Omega-m} \\
i \frac{R_{s}}{p_{r}} \frac{(m+1) \mathcal{L}_{m+1}}{\Omega-(m+1)} & -\frac{C}{p_{r}} \frac{(m+1) \mathcal{L}_{m+1}}{\Omega-(m+1)}
\end{array}\right)\left(\begin{array}{c}
\sigma_{m}\left(p_{r}\right) \\
\sigma_{m+1}\left(p_{r}\right)
\end{array}\right) .
$$

The eigenvalues are solutions of the following equation:

$$
\begin{aligned}
\Omega^{2}- & {\left[(2 m+1)+2 I_{k, s}\left(m \mathcal{L}_{m} \frac{L}{p_{r}}-(m+1) \mathcal{L}_{m+1} \frac{C}{p_{r}}\right)\right] \Omega+m(m+1) . } \\
& \cdot\left[1+4 I_{k, s}{ }^{2} \mathcal{L}_{m} \mathcal{L}_{m+1} \frac{R^{2}-L C}{p_{r}{ }^{2}}-2 I_{k, s}\left(\mathcal{L}_{m+1} \frac{C}{p_{r}}-\mathcal{L}_{m} \frac{L}{p_{r}}\right)\right]=0 .
\end{aligned}
$$

Obviously, if $\Omega$ has two split real roots, $I_{k, s}$ must satisfy

$$
\left\{1-\left[(m+1) \mathcal{L}_{m+1} \frac{C}{p_{r}}+m \mathcal{L}_{m} \frac{L}{p_{r}}\right] 2 I_{k, s}\right\}^{2}-4 m(m+1) \mathcal{L}_{m} \mathcal{L}_{m+1} \frac{R^{2}}{p_{r}^{2}} \cdot 4 I_{k, s}^{2}>0
$$

Above the transition, $\eta>0, I_{k, s}>0$, and

$$
I_{k, s_{t h}}<\frac{1}{2(m+1) \mathcal{L}_{m+1} \frac{C}{p_{r}}+2 m \mathcal{L}_{m} \frac{L}{p_{r}} \pm 2 \sqrt{m(m+1) \mathcal{L}_{m} \mathcal{L}_{m+1}} \frac{R}{p_{r}}} .
$$

The severe condition is given by taking the positive sign in the last term of the denominator. This is because in this case both the inductive and capacitive impedances help the resistive to decrease the value of the merging point. The physical meaning is clear: because the spectrum of the lower mode $(m)$ overlaps with the inductive part of the impedance, it pulls 
the coherent frequency up, and the spectrum of the higher mode $(m+1)$ overlaps the capacitive part of the impedance, and thus pulls the coherent frequency down. Together, these increase the merging speed and lower the value of the merging point.

Below transition, the situation is just the opposite: the inductive part of the impedance pulls the lower mode $(m)$ down, and the capacitive part of the impedance pulls the higher mode $(m+1)$ up, so that the merging speed decreases, and the value of the merging point is increased. It can be seen from condition (17), that because $\eta<0$ and $I_{k, s}=-\left|I_{k, s}\right|<0$, the condition becomes

$$
\left|I_{k, s}\right|_{t h}>\frac{-1}{2(m+1) \mathcal{L}_{m+1} \frac{C}{p_{r}}+2 m \mathcal{L}_{m} \frac{L}{p_{r}} \pm 2 \sqrt{m(m+1) \mathcal{L}_{m} \mathcal{L}_{m+1}} \frac{R_{s}}{p_{r}}}
$$

If the last term in the denominator takes a positive sign, the right-hand side of Eq. (19) becomes minus. This means that there is never merging together at all. However, if a minus sign is taken, and when $(m+1) \mathcal{L}_{m+1} C+m \mathcal{L}_{m} L<\sqrt{m(m+1) \mathcal{L}_{m} \mathcal{L}_{m+1}} R_{s}$, merging occurs somewhere. However, due to the opposite effects of the reactance and resistance, the value of the merging point is increased.

Although the above explanation seems to be less persuasive, it coincides with the practical case in which the net result of the upper mode $(m+1)$ interacting with the resonator impedance always appears to be more capacitive (or less inductive), compared with the lower mode $(m)$. It is thus well justified by the numerical-calculation results of Eq. (6). Figures 5(a) and 5(b) are typical examples for $Q=1$ and $K=1$.

The adjacent azimuthal modes remain separated until a very high current in the case $\alpha<0$, whereas different modes merge even at a very low intensity in the case $\alpha>0$.

In the above analysis, since we neglected the frequency spread $(\omega(J))$, the value of the merging point is just equal to the threshold of the azimuthal mode coupling. However, if we include the frequency spread, the value of the merging point is not equal to the instability threshold. However the condition of merging is a necessary, but insufficient, condition for exciting azimuthal mode coupling instabilities. Thus, in the case that $\alpha<0$, most instabilities occur between the radial modes within the same azimuthal band; however, in the case that $\alpha>0$ the mechanism of instabilities is more complex. It sometimes occurs with a mixture of the radial and azimuthal modes. Once the azimuthal mode coupling instabilities occur, the growth rate rapidly increases with the intensity. This is also shown in Figures 5(a) and 5(b).

2. From a general point of view, the threshold of microwave instabilities is higher over a rather wide range of $K$ values for the case $\alpha<0$. Even in the range where the threshold is lower than in the case $\alpha>0$, in most cases bunch lengthening is still much less serious than in the case $\alpha>0$, if the machine is operated at the same intensity.

Comparisons of the current thresholds between both $\alpha>0$ and $\alpha<0$ cases are given in Figure 6. In this comparison, the growth rate $5 \times 10^{-3}$ (in units of $\omega_{s 0}$ ) is chosen as the threshold for all unstable modes, and radiation damping is omitted.

Similar characteristics have been obtained for different $Q$, so we can take the more typical one, $Q=0.6$, as an example for a detailed description. From this picture, one can find three different regions: $K<0.75 ; 0.75<K<1.1 ; K>1.1$. 


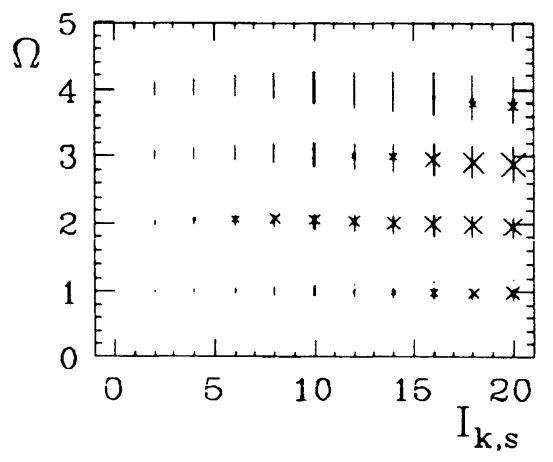

(a) $a<0$

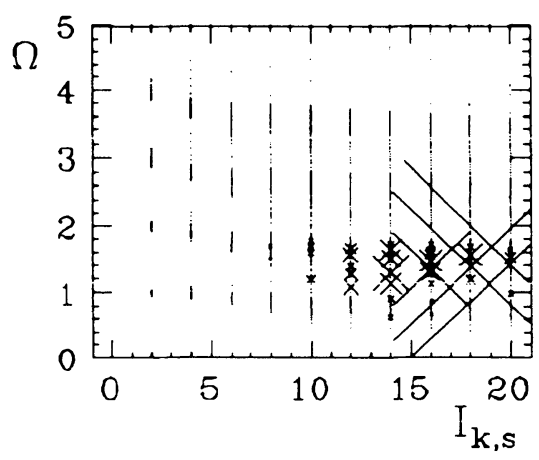

(b) $a>0$

FIGURE 5: Adjacent azimuthal modes merge with the intensity and the magnitude of the growth rate (proportional to the size of the cross).

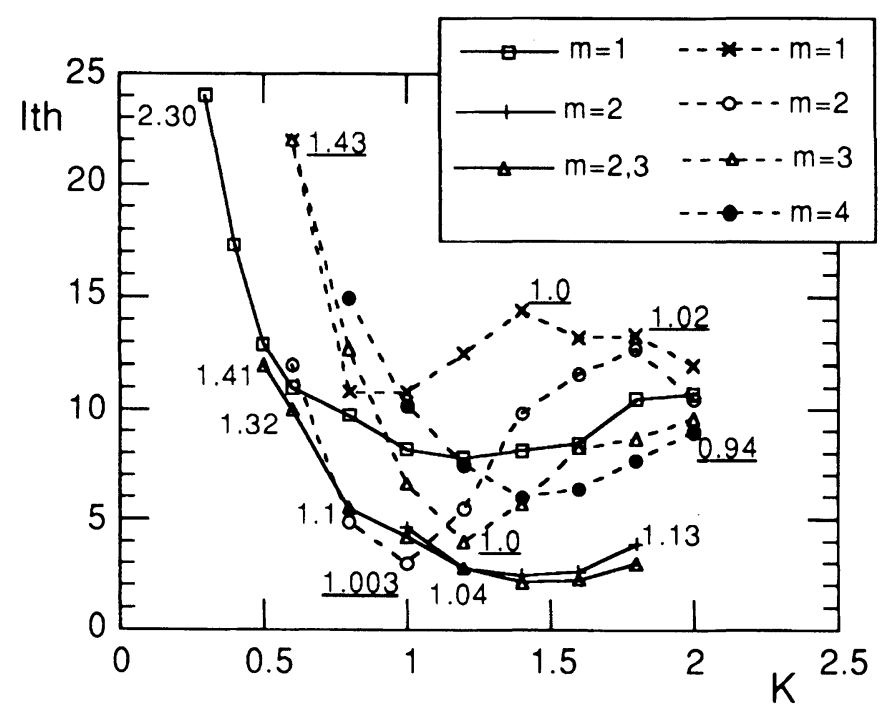

FIGURE 6: Current threshold vs $K$ for $Q=0.6$ (Solid lines for $\alpha>0$ and dashed lines for $\alpha<0$ ). The numbers attached to the marks indicate the bunch lenthening $\left(\sigma_{z} / \sigma_{z 0}\right)$, with underline for $\alpha<0$ and without that for $\alpha>0$.

1) $K>1.1$. In this region the threshold of $\alpha<0$ is higher than the threshold of $\alpha>0$, and the system appears to be inductive (or negative inductive) for $\alpha>0$ (or $\alpha<0$ ).

In the case where $\alpha<0$, since all of the azimuthal modes are fully separated, a total of four pure radial mode instabilities (each occurs in its own azimuthal band), appear in this region. All of these instabilities are not strong, and the increase in the growth rate with intensity is quite slow. For example, when $I_{k, s}=I_{k, s_{t}}+10$, the corresponding growth rate only increases five times, i.e., from $5 \times 10^{-3}$ to $2.5 \times 10^{-2}$. The lowest threshold among the four instabilities is $I_{k, s}=4$ at $K=1.2$. 
In the case where $\alpha>0$, two pure radial mode and one azimuthal mode instabilities appear in this region. The azimuthal mode instability is the strongest; it occurs in the merging region of mode 2 and mode 3 when the intensity is high. Nevertheless, its threshold is not the lowest one. The lowest threshold which becomes the boundary in this region is determined by one of the two pure radial modes which occur when azimuthal modes 1 and 2 are separated at low intensity. These two instabilities are rather weak, and the maximum growth rate is never over $2.5 \times 10^{-2}$ when the intensity is above the threshold. They finally both disappear at $K>1.8$; mode 2 also disappears at $K<1.0$. The corresponding threshold with different $K$ is lower than that in the case $\alpha<0$.

The bunch lengthening $\left(\frac{\sigma_{z}}{\sigma_{z 0}}\right)$ at the threshold is also shown in Figure 6. Obviously, the bunch lengthening is much stronger in the case $\alpha>0$.

2) $0.75<K<1.1$. The threshold of $\alpha<0$ is lower than that of $\alpha>0$; in this region the resistive character is dominant, with a little inductive (or negative inductive) character when $K>0.9$, and with a little capacitive (or negative capacitive) character when $K<0.9$ for $\alpha>0$ (or $\alpha<0)$.

In the case $\alpha<0$, although the four pure radial mode instabilities still exist, the threshold of mode 2 decreases very rapidly with decreasing $K$, and arrives at its minimum at $K=1$, giving the lowest threshold value.

For $\alpha>0$, only mode 1 still exists, and gives the boundary of the threshold when $K>0.8$. Although its value is high, bunch lengthening is very strong.

The maximum difference in the threshold value between $\alpha<0$ and $\alpha>0$ is less than $50 \%$ that at $K=1$; the former is $I_{k, s}=3$, and the latter is $I_{k, s}=4.2$. If a machine with $\alpha>0$ is operated at the same intensity as the threshold of $\alpha<0$, i.e., $I_{k, s}=3.0$, the bunch lengthening is 1.07 , which is higher than 1.001 for $\alpha<0$.

For increasing the threshold of $\alpha<0$ in this region, the simplest way is to increase the energy spread of the beam; one can roughly estimate the bunch lengthening at the new higher threshold by the scaling. A calculation shows that it is effective in most cases; only about a $5 \sim 10 \%$ energy spread increment is needed. One can thus easily increase the threshold of the $\alpha<0$ case to the same value as in the $\alpha>0$ case. In the meantime, the bunch lengthening is still less than that in the case $\alpha>0$.

3) $K<0.75$. The threshold of $\alpha<0$ is higher than that for $\alpha>0$, and the capacitive character is dominant in this region. The lowest threshold boundary in the case $\alpha<0$ is determined by the pure radial mode 2 instability; in the case $\alpha>0$ it is determined by the azimuthal mode instability, which is very strong, and the bunch shape is seriously deformed and two peaks appear.

It is surprising that the threshold is unbelievably high in this region. This may not be true for a realistic machine; first, a smaller $K$ means a weaker wake field, which may not be reasonable in practical cases. Second, because the real impedance cannot be correctly described sufficiently by the simple resonator impedance model, a small $K$ means that the system appears to be more capacitive for a short natural bunch length; it may also not be true. It was pointed out in Ref. 8 that the wake field of a realistic machine is always determined by many small discontinuities, such as bellows, masks, transitions, etc., and that most of them are inductive. It is quite different from the resonator impedance. Thus, in practice, most realistic machine parameters are located at the right-hand side of the picture i.e., $K>0.9$. 


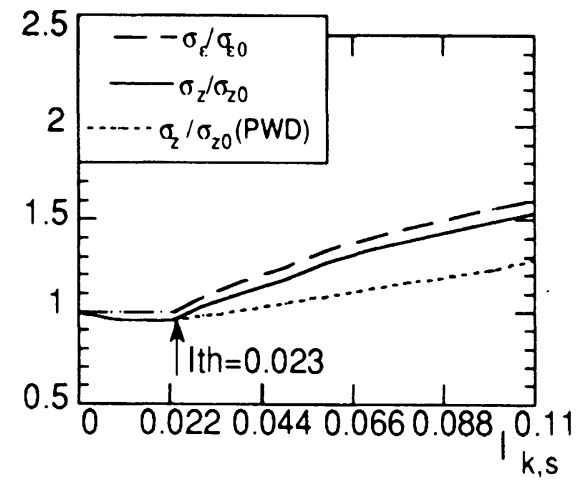

(a) $a<0$

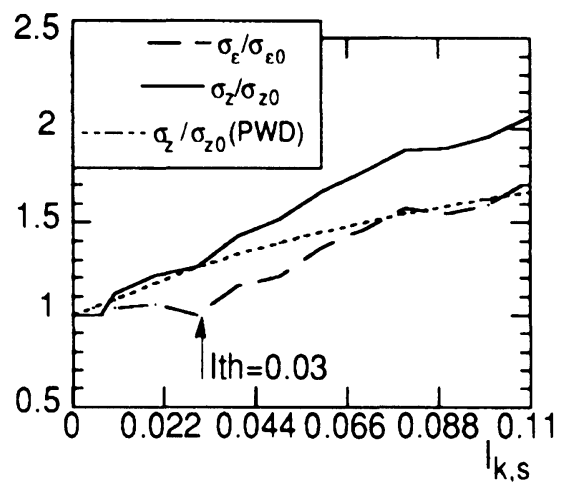

(b) $n>0$

FIGURE 7: Threshold and bunch length behaviour for $\alpha<0$ and $\alpha>0$ with the wake field of old chamber of SLC damping ring.

A real example is given here by using the wakefield of the old vacuum chamber of the SLC damping ring. Figures 7(a) and 7(b) give the thresholds for both cases; the result coincides with the analysis mentioned above.

Another very interesting phenomenon is that even above the microwave threshold the bunch length behavior is very similar to that described by a pure potential well distortion; about half the bunch lengthening is contributed by PWD in most cases.

\section{CONCLUSION}

The longitudinal bunch shape is less deformed and bunch lengthening is less serious in electron rings with negative momentum compaction factor. A natural "negative feedback" mechanism can explain the above mentioned phenomenon. Even above the threshold the intrinsic shape of the potential well field plays an important role.

\section{ACKNOWLEDGEMENTS}

The author, Dr. Fang, would like to thank Dr. Alex. Chao for his valuable discussions and help, Dr. L.C. Teng for encouragement, Dr. K.Y. Ng, Dr. T. Suzuki, Dr. Y.H. Chin, Dr. R. Baartman, Mr. M. D'yachkov for their discussions, and Dr. Y. Kimura, Dr. S. Kurokawa for their strong support and encouragement. Special thanks are given to Dr. K.L. Bane for his kindness to provide the old vacuum chamber wakefield of the SLC damping ring. Thanks also go to Dr. G.X. Li for his collaboration during the early stage. 


\section{REFERENCES}

1. A.W. Chao, Physics of Collective Beam Instabilities in High Energy Accelerator (Wiley, New York, 1993).

2. Z.Y. Guo et al., the Fifth JAPAN-CHINA JOINT SYMPOSIUM on Accelerators for Nuclear Science and Their Application, 18-20 Oct. 1993. OSAKA, JAPAN, pp. 2-6.

3. Q. Qin, Z.Y. Guo, C. Zhang, Workshop on Beam Instabilities in Storage Ring, 24-30 July 1994, Hefei, China.

4. G.X. Li, Design of BEPC Mini- $\beta$ Schemes and Research to the Bunched Beam Longitudinal Instability with negative momentum compaction factor, $\mathrm{Ph}$. D Desertation, IHEP, CAS, June 1993.

5. K. Oide and K. Yokoya, KEK-preprint 90-10 (1990); K. Oide, KEK preprint 94-138 (1994); K. Oide, in Nonlinear Dynamics and Particle Acceleration, AIP Conf. Proc. 230, p. 266 (1990).

6. K. Yokoya, Bunch Lengthening above Threshold by Quasi-linear Theory, KEK preprint, Nov. 1994.

7. B. Chen, The Longitudinal Collective Instabilities of Nonlinear Hamiltonian Systems in Circular Accelerators, $\mathrm{Ph}$. D Desertation, Oct. 1994.

8. K.L. Bane and K. Oide, Proceeding of the 1993 Particle Accelerator Conference, Washington D.C., 17-20 May 1993, (1993) p. 3339. 